\title{
Automated segmentation and detection of T1-weighted magnetic resonance imaging brain images of glioma brain tumor
}

\author{
Nur Hanina Izani Muhammad Zaihani' ${ }^{1}$, Rosniza Roslan², Zaidah Ibrahim³, \\ Khyrina Airin Fariza Abu Samah ${ }^{4}$ \\ ${ }^{1,2,4}$ Faculty of Computer and Mathematical Sciences, Universiti Teknologi MARA Melaka Kampus Jasin, Malay sia. \\ ${ }^{3}$ Faculty of Computer and Mathematical Sciences, Universiti Teknologi MARA, Malay sia
}

\begin{tabular}{|c|c|}
\hline Article Info & ABSTRACT \\
\hline Article history: & \multirow{10}{*}{$\begin{array}{l}\text { There are numerous studies on brain imaging applications. The statistics } \\
\text { in Malaysia showed that glioma is one of the most common type disease } \\
\text { in brain tumor. Glioma brain tumor is an abnormal growth of glial cells inside } \\
\text { the brain tissues which known as cerebral tissues. Radiologist commonly used } \\
\text { magnetic resonance imaging (MRI) image sequences to diagnose the brain } \\
\text { tumor. However, manual examination of the brain tumor diagnosis by } \\
\text { radiologist is difficult and time-consuming task as tumors are occurred in } \\
\text { variability of shape and appearance. They will also inject a gadolinium contrast } \\
\text { agent to enhance the image modality which will give the side effects to } \\
\text { the patients. Therefore, this paper presents an automated segmentation } \\
\text { and detection of MRI brain images using Sobel edge detection } \\
\text { and mathematical morphology operations. The total of } 30 \text { glioma T1-weighted } \\
\text { MRI brain images are obtained from brain tumor image segmentation } \\
\text { benchmark (BRATS). The results of segmentation and detection } \\
\text { are quantitatively evaluated by using Area Overlap which produced } \\
\text { the accuracy rate of } 80.2 \% \text { and shows that the presented methods } \\
\text { are promising. }\end{array}$} \\
\hline Received Oct 18, 2019 & \\
\hline Revised Dec 28, 2019 & \\
\hline Accepted Feb 17, 2020 & \\
\hline Keywords: & \\
\hline Detection & \\
\hline Glioma brain tumor & \\
\hline MRI brain images & \\
\hline Segmentation & \\
\hline T1-weighted & \\
\hline
\end{tabular}

This is an open access article under the $\underline{C C B Y-S A}$ license.

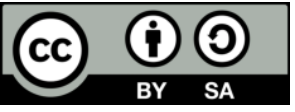

\section{Corresponding Author:}

Nur Hanina Izani Muhammad Zaihani,

Faculty of Computer and Mathematical Sciences,

Universiti Teknologi MARA Melaka Kampus Jasin,

77300 Merlimau, Melaka.

Email: ninaizanizaihani@gmail.com

\section{INTRODUCTION}

There are numerous studies in brain imaging domain such as brain abnormalities detection, brain mapping, brain stroke analysis, brain tumor volume analysis, brain tissue classification, epilepsy analysis, and brain tumor segmentation in a near future [1-8]. Due to the advancement and innovation of digital media technologies, these brain imaging applications are beneficial to the patients that experience with the brain abnormalities problem. Nowadays, there are several methods that can be applied in brain tumor applications such as image processing, deep learning, mathematical modeling and genetic algorithm [3-16]. However, it is challenging task in image processing when to segment the brain tumor as the homogeneity intensity of tumor, cerebral and non-cerebral tissues. Therefore, this paper employed on the structural imaging tool of T1-weighted Magnetic Resonance Imaging (MRI) to segment and detect the glioma brain tumor rapidly. MRI tool provides the higher-definition images for the soft tissues of brain tissues [1] and it is helpful in examining the brain tissues and location of the brain tumor tissues. T1-weighted MRI brain images are produced from the short repetition time (TR) and each time (TE). The contrast and brightness 
of the image are predominately determined by $\mathrm{T} 1$ properties of the tissues. Glioma brain tumor refers to the abnormal growth in the brain [2] which is the abnormal growth of glial cells within the brain. The abnormal growth varies based on the patient type and condition. Brain tumor can be classified as primary and secondary (metastatic) tumor. The primary tumor comes from the cells that originally from the brain tis sue cells while metastatic tumor is cancerous cells which comes from the other part of the body that are spread to the brain [4, 17-20]. The glioma brain tumor can be graded from low-grade such as astrocytomas and oligodendrogliomas to high-grade glioma such as glioblastoma multiform [4]. Therefore, it is advantageous to the patient if they performed an early treatment of the brain tumor identification.

Image segmentation is a vital task in image processing and performed at the low-level image processing phase [20, 21]. It is defined as partitioning an image into a homogenous region area [5, 12]. Brain tumor segmentation refers to the partition and segment the brain tumor region area. There are several techniques that have been used for image segmentation such as edge detection and morphological operations technique. A lot of previous studies in image segmentation showed that region-based methods produced the higher accuracy results. However, a small amount of researchers works on the automated brain tumor segmentation. There are several of image segmentation techniques that can be applied to segment the region area such as watershed edge detection methods, thresholding, region growing, split and merge and mathematical morphology [22]. Edge detection is one of the most widely approach that can be applied in detecting the edges within the image. It works based on detecting abrupt local changes in intensity of image. Edge pixels, refers to an intensity pixel of an image function changed abruptly. The earliest operator in the field of edge detection is Roberts cross-gradient operator [23]. It computes the gradient image by using 2D masks and gives preference to diagonal edges. Mathematical morphology operations are used to identify the shape, structure, edges and holes from the binary image type $[1,4]$. There are four basic mathematical morphology operations which are dilation, erosion, opening and closing that operate with the structuring element. The erosion operation is employed to intrigued segment of the image by utilizing the structuring components [24]. The undesirable part is expelled and only the intrigue part is shown.

This paper presents an automated segmentation and detection of T1-weighted MRI brain images of glioma brain tumor using the combination of Sobel edge detection and mathematical morphological operations. This paper is comprised as follows. Section 1 clarifies the motivation and existing research on brain imaging research. Section 2 discuss on the process flow of brain segmentation, detection and the data that conducted during this research. This section is also describes the details on methods and experimental techniques used in the experiments. Section 3 analyses the experimental results and evaluation that have done in the experimental process. The conclusion and recommendation for future works are explain in the Section 4.

\section{RESEARCH METHOD}

The main objective of this paper is to automate segment and detect the brain tumor of T1-weighted MRI brain images of axial orientation. There are three phases that have been conducted during the experiment which are data collection, processing (i.e. brain tumor segmentation and detection) and post-processing (i.e. test and evaluation). Figure 1 shows the process flow of the proposed algorithm for segmentation and detection.

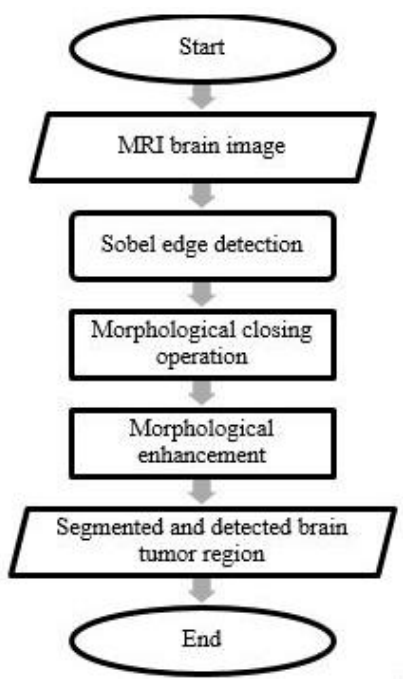

Figure 1. Flowchart of proposed algorithm 


\subsection{MRI image dataset}

MRI T1-Weighted of two-dimensional brain images has been collected from the online dataset, brain tumor image segmentation benchmark (BRATS). The experimented glioma T1-weighted image sequence of axial orientation has been utilized in this paper. The total number of images experimented are from 30 brain images. The experimented glioma brain tumor refers to the brain tumor tissues which excluding the oedema part. Brain oedema is defined as the brain water content within the brain images which leads to increase in tissues volume [18]. Figure 2 shows the sample of glioma of T1-weighted MRI brain images.

Brain Tumor Area

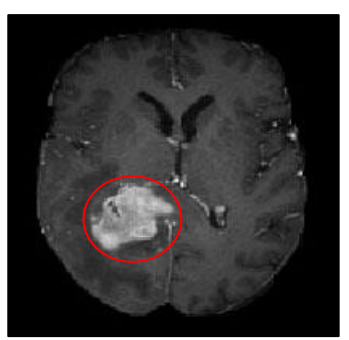

Figure 2. Sample of T1-weighted MRI brain image

\subsection{Segmentation and detection}

The input image of MRI brain image is converted into a grayscale image as shown in Figure 3. Then the Sobel edge detection has been performed on the grayscale image. Sobel edge detection is a technique which to discover edges utilizing the Sobel approximation to the derivative. It returns edges at those points where the angle of 1 is most extreme. The result image of Sobel edge detection is applied as shown in Figure 3(b). The morphological operations of closing is then utilized from the binary image of Sobel edge detection result. The closing operation is applied on dilation operation and then followed by the erosion operation. This paper experimented on a disk-shaped structuring element with a radius of 3 pixels. Equation (1) for dilation and (2) for erosion operations [25] are represented as follows.

Dilation:

$$
A \oplus B=\left\{z \mid\left(B^{\wedge}\right)_{z}: \cap A \neq \emptyset\right\}
$$

where:

$$
\begin{array}{lll}
\emptyset & = & \text { empty set } \\
\mathrm{z} & = & \text { set of all points in } \mathrm{A}
\end{array}
$$

Erosion:

$$
A \ominus B=\left\{z \mid(B)_{z} \cap A^{C} \neq \phi\right\}
$$

where:

$$
\begin{array}{lll}
\phi & = & \text { empty set } \\
\mathrm{z} & = & \text { set of all points in } \mathrm{A}
\end{array}
$$

The dilation A by B of (1) is a set which consists of all the structuring elements origin location where $\mathrm{B}$ elements are reflected and translated with the overlaps of at least one element of $\mathrm{A}$. The erosion of (2) indicates that the erosion of A by B is a set of all structuring elements origin location where the translated B elements has no overlap with the background of A. Figure 3(a)-(g) shows the sample result of the Sobel edge detection and mathematical morphology operations.

The MRI brain grayscale input image is experimented using Sobel edge detection method. The morphological closing operation is applied on the result image of the Sobel edge detection. The largest area of the MRI brain image is computed and the other small areas are removed. Then, morphological enhancement is used to fill-in any detected holes of MRI brain image. The mathematical morphology operations of erosion and dilation are performed. This experiment utilized a sphere-shaped structuring element with a radius of 2 pixels for the morphological erosion operation and a disk-shaped structuring element with a radius of 6 pixels for the morphological dilation operation. By applying these morphological operations, 
the unwanted region is removed and the glioma brain tumor affected region is displayed. Figure 3(f) shows the sample of automated segmentation and detection result of the glioma brain tumor image.

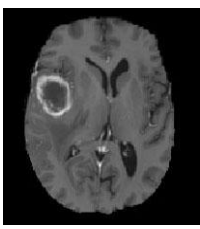

(a)

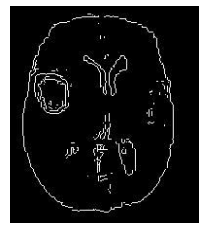

(b)

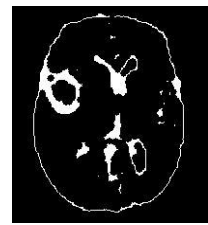

(c)

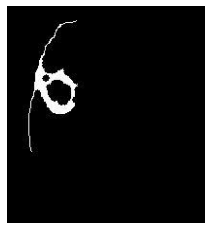

(d)

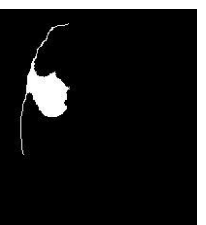

(e)

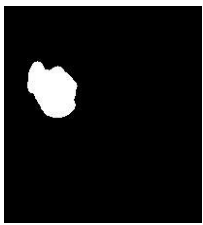

(f)

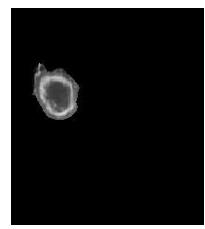

(g)

Figure 3. The process of MRI brain image segmentation and detection, (a) Original image, (b) Result of sobeledge detection image, (c) Morphological closing operation, (d) Largest region, (e) Morphological enhancement using fill-in holes, (f) Segmented of brain tumor image,

(g) Detected area of glioma brain tumor

\section{RESULTS AND DISCUSSION}

The quantitative evaluation is evaluated in this experiment for T1-weighted MRI brain images of axial orientation. The segmented MRI brain images are computed using Area Overlap (AO). The ground truth image data is created and generated by BRATS database to set as a benchmark which to compute the AO. AO is one of the method to measure the overlapping percentage area between the MRI brain tumor area and ground truth area $[1,2,20]$. AO accuracy rate is calculated by using the following (3).

$$
A O=100 \times \frac{\left|X_{1} \cap X_{2}\right|}{\left|X_{1} \cup X_{2}\right|}
$$

where

$\mathrm{X}_{1}$ represents the area of the proposed segmented MRI brain tumor image

$\mathrm{X}_{2}$ represents the area of the ground truth data

Based on the 30 images of T1-weighted MRI brain images, the average result of the AO accuracy rate is $80.2 \%$. Table 1 illustrates the sample results of the proposed segmented MRI brain tumor image.

Table 1. Sample results of segmented MRI T1-weighted glioma brain tumor image

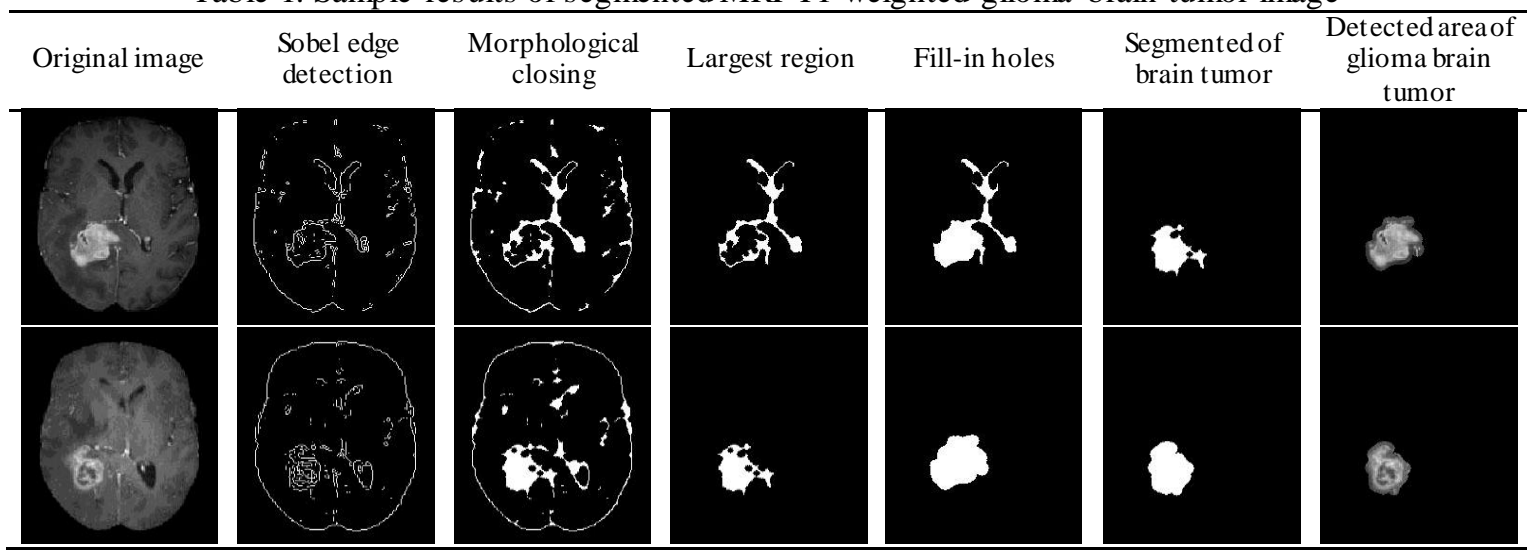

\section{CONCLUSION}

This paper presents an automated segmentation and detection of T1-weighted MRI brain images of glioma brain tumor using the combination of Sobel edge detection and mathematical morphological operations. Therefore, this paper experimented on the Sobel edge detection and mathematical morphology operations of closing, dilation, erosion and filling holes. The quantitative evaluation using area overlap 
of the segmented MRI glioma brain images are produced $80.2 \%$ of the average accuracy rate. It is showed that Sobel edge detection and mathematical morphology methods produced the promising result of MRI brain tumor since it is challenging task which to avoid using the contrast enhanced MRI brain tumor image. Thus, it is also beneficial and assist the radiologist in early detection of glioma brain tumor. This research significantly for the automated detection of the glioma MRI brain tumor in an early stage and it may give chances to the patients for a treatment. This paper is also highlight on the employment of the T1-weighted MRI brain images. It demonstrates that advantageous of this research which focuses on the T1-weighted is easy for the radiologist to identify the location of tumor within MRI brain image. Besides, this proposed research may use for the other segmentation and detection of the brain abnormalities. For the future works, future research to prevent in segmenting similar intensity of brain tissues within the image. The further work may also concentrate on the identification and recognizing the other types of brain abnormalities and their analysis.

\section{ACKNOWLEDGEMENTS}

The authors would like to thank the Ministry of Higher Education, Malaysia and Universiti Teknologi MARA for the research funding and support via grant number FRGS/1/2017/ICT05/UITM/03/1.

\section{REFERENCES}

[1] R. Roslan, N. Jamil, and R. Mahmud, "Skull stripping magnetic resonance images brain images: region growing versus mathematical morphology," International Journal of Computer Information Systems and Industrial Management Applications, vol. 3, pp. 150-158, 2011.

[2] R. Roslan, N. Jamil, and R. Mahmud, "Skull stripping of MRI brain images using mathematical morphology," IEEE EMBS Conference on Biomedical Engineering and Sciences (IECBES), pp. 26-31, 2010.

[3] M. Mittal, L. M. Goyal, S. Kaur, I. Kaur, A. Verma, and D. J. Hemanth, "Deep learning based enhanced tumor segmentation approach for MR brain images," Applied Soft Computing, vol. 78, pp. 346-354, 2019.

[4] D. Haritha, "Comparative study on brain tumor detection techniques," International Conference on Signal Processing, Communication, Power and Embedded System (SCOPES), pp. 1387-1392, 2016.

[5] A. Işın, C. Direkoğlu, and M. Şah, "Review of MRI-based brain tumor image segmentation using deep learning methods," Procedia Computer Science, vol. 102, pp. 317-324, 2016.

[6] M. Sharma and S. Mukherjee, "Fuzzy C-means, ANFIS and genetic algorithm for segmenting astrocy toma-a ty pe of brain tumor," International Journal of Artificial Intelligence, vol. 3, no. 6, pp. 16-23, 2014.

[7] P. S. Duth, V. Viswanath, and P. Sreekumar, "Brain image segmentation using level set: An hybrid approach," International Journal of Advances in Applied Sciences , vol. 6, no. 3, pp. 267-276, 2017.

[8] N. S. Noor, N. M. Saad, A. R. Abdullah, and N. M. Ali, "Automated segmentation and classification technique for brain stroke," International Journal of Electrical and Computer Engineering, vol. 9, no. 3, pp. 1832-1841, 2019.

[9] K. A. Nawas, M. Mustafa, R. Samad, D. Pebrianti, and N. R. Abdullah, "K-NN classification of brain dominance," International Journal of Electrical and Computer Engineering, vol. 8, no. 4, pp. 2494-2502, 2018.

[10] S. L. Bangare, G. Pradeepini, and S. T. Patil, "Neuroendoscopy adapter module development for better brain tumor image visualization," International Journal of Electrical and Computer Engineering, vol. 7, no. 6, pp. 3643-3654, 2017.

[11] M. Hamiane and F. Saeed, "SVM classification of MRI brain images for computer-assisted diagnosis," International Journal of Electrical and Computer Engineering, vol. 7, no. 5, pp. 2555-2564, 2017.

[12] G. R. Chandra and K. R. Rao. "Tumor detection in brain using genetic algorithm," Procedia Computer Science, vol. 79, pp. 449-457, 2016.

[13] N. Menon, M. Karnan, and R. Sivakumar, "Brain tumor segmentation in MRI image using unsupervised artificial bee colony and FCM clustering," International Journal of Computer Science and Management Research, vol. 2, no. 5, pp. 2450-2454, 2013.

[14] S. Roy, S. Nag, I. K. Maitra, and S. K. Bandyopadhyay, "A review on automated brain tumor detection and segmentation from MRI of brain," International Journal of Advanced Research in Computer Science and Software Engineering, vol. 3, no. 6, pp. 1706-1746, 2013.

[15] O. Salima, A. Taleb-Ahmed, and B. Mohamed, "Spatial information based image clustering with a swarm approach," International Journal of Artificial Intelligence, vol. 1, no. 3, pp. 149-160, 2012.

[16] M. Prastawa, E. Bullitt, S. Ho, and G. Gerig, "A brain tumor segmentation framework based on outlier detection," Medical Image Analysis, vol. 8, no. 3, pp. 275-283, 2004.

[17] V. Vijay, A. R. Kavitha, and S. R. Rebecca, "Automated brain tumor segmentation and detection in MRI using enhanced Darwinian particle swarm optimization (EDPSO)," Procedia Computer Science, vol. 92, pp. 475-480, 2016.

[18] S. Mahajan and H. Bhagat, "Cerebral oedema: Pathophysiological mechanisms and experimental therapies," Journal of Neuroanaesthesiology and Critical Care, vol. 3, no. 4, pp. 22, 2016.

[19] R. Roslan and N. Jamil, "Texture feature extraction using 2-D Gabor filters," International Symposium on Computer Applications and Industrial Electronics (ISCAIE), pp. 173-178, 2012. 
[20] R. Roslan, N. Jamil, and N. Za'ba, "Spectral texture segmentation for glioma brain tumour detection, ”Journal of Next Generation Information Technology, vol. 4, no. 6, pp. 74-85, 2013.

[21] F. Fitrianingsih, S. Madenda, E. Ernastuti, S. Widodo, and R. Rodiah, "Cursive handwriting segmentation using ideal distance approach," International Journal of Electrical and Computer Engineering, vol. 7, no. 5, pp. 2863-2872, 2017.

[22] B. Bhatkalkar, A. Joshi, S. Prabhu, and S. Bhandary, "Automated fundus image quality assessment and segmentation of optic disc using convolutional neural networks," International Journal of Electrical and Computer Engineering, vol. 10, no. 1, pp. 816-827, 2020.

[23] A. Aslam, E. Khan, and M. S. Beg, "Improved edge detection algorithm for brain tumor segmentation," Procedia Computer Science, vol. 58, pp. 430-437, 2015.

[24] S. M. Aung, K. Kanokwiroon, T. Phairatana, and S. Chatpun, "Live and dead cells counting from microscopic trypan blue staining images using thresholding and morphological operation techniques," International Journal of Electrical and Computer Engineering, vol. 9, no. 4, pp. 2088-8708, 2019.

[25] Gonzalez RC, Woods RE. Digital Image Processing, Third Edition. Pearson, 2007. 